\title{
Green Chemistry: An Enabler for Using Thorium as Green Energy Fuel
}

\author{
Ruken Esra Demirdogen ${ }^{1}$, Tunay Kontas Askar ${ }^{2}$ \\ ${ }^{1}$ CankiriKaratekin University, Department of Chemistry, Faculty of Science,Cankiri 18200, Turkey \\ ${ }^{2}$ CankiriKaratekin University, Department of Chemistry, Faculty of Science, Cankiri 18200, Turkey
}

\begin{abstract}
The current trends of life styles, growth, consumption and production put greater emphasis on the importance and urgency of producing massive quantities of energy. The depleting nature and the accelerated demand of commercial energy force planners and policy makers to look for alternative sources. Therefore, clean energy, environmentally benign and economical sources are being sought. Because environmental problems stem from the use of fossil fuels and the renewable energy sources are not sufficient with respect to their energy contents and intrinsic nature, nuclear energy is considered as a possible source of energy in the world. In this respect, Thorium attracts much attention as it is the Nuclear Energy fuel with the least negative consequences such as radioactivity and nuclear proliferation. However, the strict regulations and the lack of an eco-friendly monitoring and analysis method besides the doubts about the greenness of Thorium stands as important obstacles on its way to become a widely used Nuclear Energy fuel. In this review paper, we aimed to give information about thorium as an effective and efficient green energy source according to the principles of green chemistry perspective.
\end{abstract}

Keywords: green chemistry, thorium, green energy

\section{Introduction}

Green Chemistry (GC) is a science-based, non-regulatory, economically driven approach toward Sustainable/ Green Development and is the use of chemistry techniques and methodologies that reduce or eliminate the use or generation of feed-stocks, products, byproducts, solvents, reagents, etc. that have adverse effect on human health or the environment [1]. The ever increasing world population, which is expected to rise from 3.3 billion people to $8-10$ billion people by 2050 , and the demand for social, economical and industrial development require and acquire production of massive amounts of energy. Energy is inevitable for human life and a secure and accessible supply of energy is crucial for the sustainability of modern societies. The world heavily relies on fossil fuels to meet its energy requirements-fossil fuels global energydemands. Use of fossil fuels is set to face such as oil, gas and coal are providing almost $80 \%$ of the multiple challenges: depletion of fossil fuel reserves, global warming and other environmental concerns, geopolitical and military conflicts and of late, continued and significant fuel price rise. These problems indicate an unsustainable situation [2].

It can be seen in Fig 1 that if the current style of production and consumption are not abandoned, fossil fuels will continue to be the major energy source [3]. This situation when accompanied by supply crises would create an ecounfriendly and insecure energy future which would be detrimental to sustainable development.

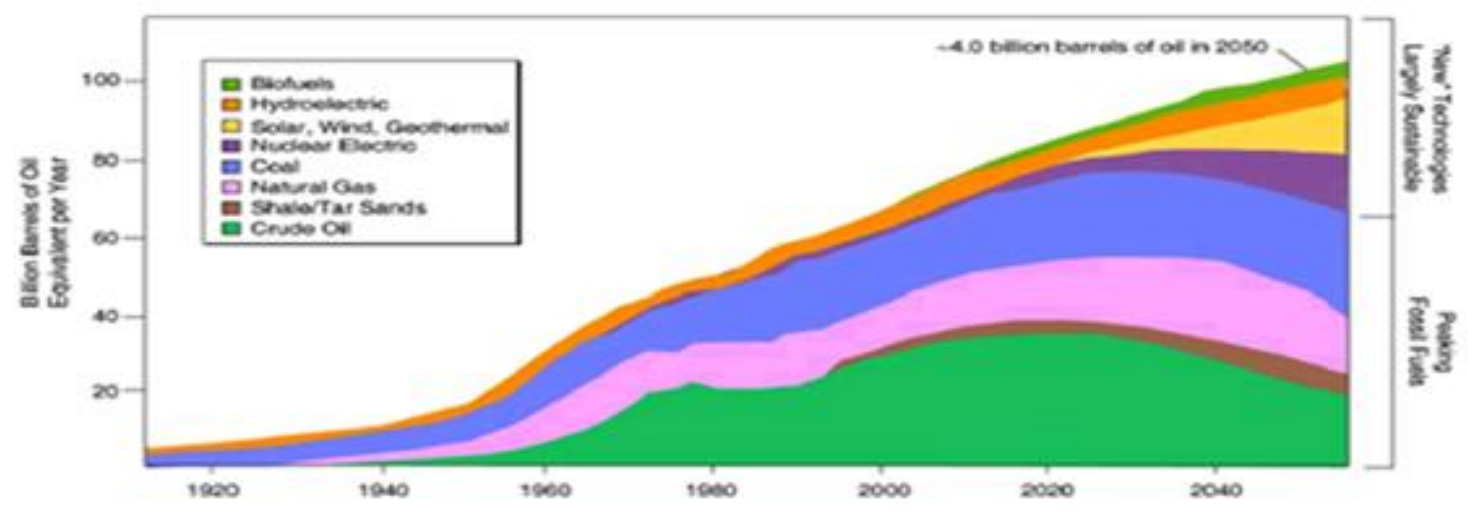

Figure 1: World energy demand with respect to long-term energy sources [3]

Renewableenergy is the solution to the growing energy challenges. Renewable energy resources such as solar, wind, biomass, and wave and tidal energy, are abundant, inexhaustible and environmentally friendly.Besides this, in the past decades have shown direct correlation between economic growth and energy usage and the current economic system demands exponential increase in energy
production.These findings indicate that the cost of meeting the necessary energy demand should be minimized. Therefore, the energy demand should be met only by using emission-free or as called 'green energy sources' such as renewables (wind, solar, hydro, etc.) and nuclear [1],[2].

According to twelve principles of green chemistry, 


\section{International Journal of Science and Research (IJSR) \\ ISSN (Online): 2319-7064}

Index Copernicus Value (2015): 78.96 | Impact Factor (2015): 6.391

greenness of an energy source lies in its capability of providing the required quantity of energy with sufficiently low cost, high feasibility and without encroaching on ecology. This requires efficient usage of GC. Although green energy sources are recalled to be renewable energy sources, not all renewable sources meet the criteria set out in the principles of GC which constitute the basis for evaluation of greenness of energy sources. Apart from hydro power in the few places, where it is plentiful, none of the renewable energy sources is sufficient to meet neither the current nor the future energy demand. They are neither intrinsically (i.e., unpredictable, intermittent nature and comparatively low energy content) nor economically suitable for large-scale power generation especially when continuous, reliable energy supply is needed [1].

Without nuclear power (NP) the world would have to rely almost entirely on fossil fuels, especially on coal, to meet the demand for base-load electricity production. Any evaluation of alternative fuels and their contribution to climate change, ecologic degradation must include consideration of "energy density", which is a measure of the amount of energy stored in a given volume or mass (weight) of that fuel, and should not be based on speculations which end up in yes/no dichotomy [4]. Taking the world energy demand into consideration it is clear that a combination of all emissionfree energy sources, such as nuclear, hydropower and other renewables (wind, solar, etc.) should be used [1].

Nuclear power is the main source of energy in some of the major developing countries in the world and contributes to almost $7 \%$ of the global energy demands. Nuclear energy (NE) has played a major role in reducing the world's use of oil for electricity generation over the past three decades. Nuclear power is only contributing $6.5 \%$ of the total energy needs. In 2003, there were 440 nuclear power plants operating in 31 countries around the world Although not every reactor is producing electricity [2].

In nuclear power reactors (NPRs) as U fissions plutonium $(\mathrm{Pu})$, which is an essential ingredient for producing nuclear bomb, is obtained [5]. In a nuclear weapon the chain reaction is very critical since extreme amounts of $\mathrm{Pu}$ or $\mathrm{U}$ which is beyond critical, are produced. Energy is extremely rapidly released and this results in a massive explosion. Whereas, in a NPR, the reaction is far slower and more controlled. However, the possibility of nuclear weapon production has lead to a never ending controversy regarding nuclear proliferation and intensified by the drawback of the radioactive character of NE. On the other hand, due to great energy requirement of the world and the advantages NP offers, NE could never be excluded from the energy agenda of the world. The rising costs of natural gas and coal, together with energy supply security, environmental constraints and the energy density of NE fuels have been the key factors which contributed to the growth in the demand or NE. Besides this exploitation of eco-friendlier or greener energy fuels would render NE more attractive [6].

\section{Thorium (Th): A Green Energy Fuel?}

Like Uranium $\left({ }^{238} \mathrm{U}\right),{ }^{232} \mathrm{Th}$ is not fissile itself, but it is fertile. It cannot directly be used in a thermal neutron reactor, but after absorbing slow neutrons it goes through two beta decays and produces ${ }^{233} \mathrm{U}$ which is fissile. In other words, it needs a fissile material such as U-233, U-235 or Plutonium (Pu-239) as a 'driver' for to maintain a chain reaction and surplus neutrons [7].

Both thorium and uranium-238 require two neutrons to release their energies. One neutron converts them into a fissile form and the other neutron actually causes the fission. Thorium absorbs a neutron and becomes uranium-233, which will fission when struck by another neutron. Uranium233 is only produced from thorium [7].

In fresh Th fuel, all of the fissions (thus power and neutrons) derive from the driver component. As the fuel operates the U-233 content gradually increases and it increasingly contributes more and more to the power output of the fuel. The ultimate energy output from U-233, which is produced in breeding, depends on numerous fuel design parameters such as, fuel burn-up attained, fuel arrangement, neutron energy spectrum and neutron flux which affects the intermediate product protactinium-233 - a neutron absorber. The fission of a U-233 nucleus releases about the same amount of energy (200 MeV) as that of U-235 [7].

Thorium fuel can be chemically cleaned from the produced ${ }^{233} \mathrm{U}$ which can be recycled into new fuel or used 'in-situ' in the same fuel form. Th is a source of fissionable material from which no $\mathrm{Pu}$ or minor actinides are produced.Th fuel cycle creates ${ }^{233} \mathrm{U}$, which, if separated from the reactor's fuel, could difficultly be used for making nuclear weapons. Thus it is safer than $U$ regarding nuclear proliferation.Weaponsgrade fissionable material $\left({ }^{233} \mathrm{U}\right)$ is harder to retrieve safely and clandestinely from a thorium reactor.Therefore, Th fuel cycle is sometimes promoted as having excellent nonproliferation credentials, but it is noteworthy to mention that the USA produced about 2 tonnes of U-233 from Th during the 'Cold War', at various levels of chemical and isotopic purity, in plutonium production reactors and U-233 can be used in nuclear weapon [5]. However, the production of U233 inevitably also yields U-232 which is a strong gammaemitter, as are some decay products, making the material extremely difficult to handle and also easy to detect.

\section{Thorium Offers Marked Advantages As A Nuclear Fuel}

The advantages, which Th offers, surpass those offered by U [8]. Th-based fuels can be used to breed fissile material in slow neutron systems. This is a unique feature for Th-based fuels and is not possible with uranium fuels. Th can be used as 'fertile matrix' for fuels containing $\mathrm{Pu}$ (and even other transuranic elements like Americium). Unlike $U$ fuels no new $\mathrm{Pu}$ is produced from Th component. Therefore, the level of net consumption of this metal is rather high. This fuel provides lower levels of waste generation, less transuranic elements in the waste. Th produces 10 to 10,000 times less long-lived and less radioactive waste [9].

Of all the 26 known isotopes of Th, only 3 have half-lives sufficiently long enough to warrant a concern. After 100 to 200 years Th produces only $1 / 6^{\text {th }}$ of the actinide toxicity produced by other nuclear fuels such as by U. It is less 


\section{International Journal of Science and Research (IJSR) \\ ISSN (Online): 2319-7064 \\ Index Copernicus Value (2015): 78.96 | Impact Factor (2015): 6.391}

environmentally damaging per unit of energy extracted than other energy fuels such as coal and U. It is present in higher concentrations $(2-10 \%)$ by weight than $U(0.1-1 \%)$ in the ores and is 3 times more abundant than $U$ in the earth's crust. Thus has a longer half-life than $U$ and is much more economic. The preparation of Th fuel does not require isotopic separation, unlike the preparation of $U$ fuels. Th fuel cycle offers enormous energy security benefits in the longterm - due to its potential for being a self-sustaining fuel without the need for fast neutron reactors. It is therefore an important and potentially viable technology that seems able to contribute to building credible, long-term nuclear energy scenarios. Th mining produces a single pure isotope, whereas the mixture of natural $\mathrm{U}$ isotopes must be enriched to function in most common reactor designs [10].

The same cycle could also use the fissionable U-238 component of the natural uranium, and also contained in the depleted reactor fuel. Th cannot sustain a nuclear chain reaction without priming, so fission stops by default in an accelerator driven reactor [4], [11]. Taking into consideration the urgency of meeting the ever increasing energy demand of the world, advantages Th offers and the fact that commercial fusion power production remains unlikely before 2050the neccessity of developing the right means, which would enable exploitation of Th as NE fuel, reveals itself more each day [10].

\section{Evaluating Nuclear Energy from a Green Chemistry Perspective}

\section{GC considers design of the molecules and molecular}

transformations. One of the major constraints on NE is radioactivity. However, if energy production from thermal energy plants is to be taken into consideration the total expected radioactivity release in a 1000 MWe coal-fired power plant exposes the population to 490 person-rem/year while the release in a $1000 \mathrm{MWe}$ nuclear power plants exposes the population to 4.8 person-rem/year. The total population dose, which includes mining, reactor operation, and waste disposal, for the complete nuclear fuel cycle is only 136 person-rem/year [7]. So the main issue is the concept of invention and design. According to GC the impacts of chemical products and chemical processes should be included as design criteria. At this point GC inextricably links hazard considerations to performance criteria. According to this criteria NE exploiting Th as fuel is far better than options such as thermal plants.

2. GC does not focus only on undesirable substances that might be inadvertently produced in a process but also on all substances that are part of the process. GC recognizes that there are significant consequences to the use of hazardous substances (i.e., regulatory, handling and transport, and liability issues) besides handling of waste. In this regard there are energy producing facilities such as hydropower dams or thermal energy plants which rank above NPRs with respect to negative consequences of their functioning [1].

3. GC is applicable to all aspects of the product life cycle as well [12]. Starting with extraction until the end of the NE fuel life-cycle $\mathrm{Th}$ proves to be feasible, efficient and effective. Th which is extracted together with other rare earth elements and offers almost negligible health concern during its employment, when compared to other energy fuels like coal, uranium, etc., but offering the same amount of energy as other NE fuels and much more than that offered by coal, but can be fed back into the fuel cycle at the end of its usage period unlike others and being able to be used in the existing nuclear reactors can be said to be eco-friendlier than existing energy fuels.

4. GC is concerned about "hazard". GC is an efficient way of dealing with risk reduction and pollution prevention since in this approach the intrinsic hazards of the substances rather than those circumstances and conditions of their use that might increase their risk is of great importance. According to the definition of risk, which is the product of hazard and exposure (Risk = Hazard X Exposure), the risk of a substance depends both on the quantifiable hazard and the quantifiable exposure to that hazard [12].

\section{Green Analytical Methodology (Gam): An Enabler For Employment of Thorium For Nuclear Energy}

GAM offers techniques and methodologies that reduce or eliminate solvents, reagents, preservatives, and other chemicals that are hazardous to human health or the environment. It encompasses three key concepts:

1. To select, modify and/or develop an analytical method that would meet the specified performance criteria -the "measurement quality objectives"-so thatif an analytical data produced by it would not be able to be used would be eliminated at the beginning and thus time and money would not be wasted.

2. To use less toxic or hazardous solvents or chemicals in sample preparation and analytical measurements. When and if possible hazardous chemicals should be replaced with less hazardous chemicals. If this is not possible, then hazardous chemicals should be used in smaller amounts. This can be best achieved in sample preservation and/or preparation steps [12]. As summarized in Table 1, GAM offers many environmental, economic and social advantages. 


\section{International Journal of Science and Research (IJSR) \\ ISSN (Online): 2319-7064}

Index Copernicus Value (2015): 78.96 | Impact Factor (2015): 6.391

Table 1: Advantages offered by GAM [13].

\begin{tabular}{|c|c|}
\hline Advantagesoffered by GAM & The Means via which the advantages are provided \\
\hline \multirow{4}{*}{$\begin{array}{l}\text { Reducingconsumption } \\
\text { of reagents and solvents }\end{array}$} & Solvent free sample preparation techniques \\
\hline & Use of 'green' media \\
\hline & Reducing the scale of determinations \\
\hline & Direct analytical techniques \\
\hline \multirow{3}{*}{ SavingEnergy } & Shortening the duration of the analytical cycle \\
\hline & Application of factors enhancing the efficiency of some operations and processes \\
\hline & Catalysts and biocatalysts \\
\hline \multirow{3}{*}{$\begin{array}{l}\text { Shortening the time thatelapsesbefore real- } \\
\text { time }\end{array}$} & Results become available \\
\hline & Use of direct techniques (in-line systems) \\
\hline & Fast tests and biotests \\
\hline \multirow{3}{*}{ Reducinggas and vaporemissions } & Hermetization of analytical operations and processes \\
\hline & Solvent free sample preparation \\
\hline & Reducing the scale of determinations \\
\hline \multirow{5}{*}{ Reducingamounts of wastes and effluents } & Solvent free sample preparation techniques \\
\hline & Use of direct techniques \\
\hline & Use of reagents with a high degree of purity \\
\hline & Reducing the scale of determinations \\
\hline & Recycling of media (after clean-up) \\
\hline \multirow{5}{*}{ Reducingprofessionalexposure } & Solvent free sample preparation techniques \\
\hline & Automation of analytical processes \\
\hline & Miniaturization of monitoring instrumentation \\
\hline & Hermetization of analytical operations and processes \\
\hline & Results become available \\
\hline
\end{tabular}

3. To decrease the amount of time and/or energy required to perform an analysis. Thus, smaller samples should be used or in-situ measurements should be made. For this purpose, the method can be made more sensitive so less sample would be needed for analysis and a method requiring sample preparation (e.g., atomic absorption) can be replaced with insitu analysis by (e.g., x-ray fluorescence).

Taking into consideration the urgency of meeting the ever increasing energy demand of the world, the advantages Th offers and the fact that commercial fusion power production remains unlikely before 2050, the necessity of developing the right means which would enable exploitation of Th as NE fuel, reveals itself more each day [10]. In this respect, there is urgent need for finding effective means which would enable employment of Th as an efficient energy fuel.

\section{Conclusion}

A few decades ago, the competition between oil and nuclear energy took place directly in power generation and indirectly in end-use markets between electricity and oil products. Finally, nuclear technology was getting ready to take its share in power generation [14]. In this respect, Th attracts much attention as it is the NE fuel with the least negative consequences such as radioactivity and nuclear proliferation. However, the strict regulations and the lack of an eco-friendly monitoring and analysis method besides the doubts about the greenness of Th stands as important obstacles on its way to become a widely used NE fuel. In this study, upon being evaluated according to the principles of green chemistry perspective it is shown that when the necessary precautions are taken $\mathrm{Th}$ is an effective and efficient green energy source. The principles of green analytical chemistry can serve for the purpose of meeting the necessary requirements for exploitation of Th.

\section{References}

[1] C.Jimenez-Gonzalez, DJC. Constable, Green Chemistry and Engineering: A Practical Design Approach, A John Wiley \& Sons, Inc, Publications, New Jersey, 2011.

[2] M. Asif, T. Muneer, "Energy supply, its demand and security issues for developed and emerging economies," Renewable and Sustainable Energy Reviews, vol. 11,pp.1388-1413, 2007.

[3] Anonymous, "Expensive energy", Available: https://oneinabillionblog.com/summary2/collapse/expensive-energy.[Accessed: Jun. 28,2013]

[4] EIA International Energy Outlook 2016. DOE/EIA0484,2016

[5] OECD Uranium 2014: Resources, Production and Demand. NEA No :7209, 2014.

[6] DR. Schilling, "Is Green Energy Really Clean Energy?" Available: www.industrytap.com/green-energy-reallyclean-energy/41096. [Accessed : Mar. 01,2017]

[7] DR. Schilling,"Is Thorium the safer, Cleaner, More Powerful Alternative to Fossil Fuels?"Available: www.industrytap.com/thorium-safer-cleaner-powerfulalternative-fossil-fuels/21317. [Accessed : Jul.14,2014.]

[8] R. Martin,“Uranium Is So Last Century - Enter Thorium, the New Green Nuke.”Available:http://www.wired.com/2009/12/ff_ne w_nukes/[Accessed :Dec.21,2009]

[9] Anonymous, "Thorium" Available:http://www.worldnuclear.org/information-library/current-and-futuregeneration/thorium.aspx[Accessed : Feb.01,2017]

[10]D. Warmblash"Thorium Power Is the Safer Future of NuclearEnergy"Available:http://blogs.discovermagazine .com/crux/2015/01/16/thorium-future-nuclear-energy [Accessed :Jan.16,2015.]

[11] HH. Rogner, A. Popescu, "An introduction to energy", In:World Energy Assessment: Energy And The Challenge Of Sustainability.J. Goldemberg pp:30-37, 2000 . 


\section{International Journal of Science and Research (IJSR) \\ ISSN (Online): 2319-7064}

Index Copernicus Value (2015): 78.96 | Impact Factor (2015): 6.391

[12] Ş. Askar, Integration of health, environment and security. Nobel Publ., Ankara, 2016.

[13] KMV. Jayaram, An Overview of World Thorium Resources, Incentives for Further Exploration and Forecast for Thorium Requirements in the Near Future. In: Thorium-BasedNuclearFuel:CurrentStatus And Perspectives, IAEA-TECDOC-412 18(4):7-20, 1987.

[14]FL.Toth, HH. Rogner,“Oilandnuclear power : Past, present, and future.”En. Eco., vol. 28, pp.1-25, 2006.

Volume 6 Issue 7, July 2017 www.ijsr.net 